\title{
Klassenführung auf der Sekundarstufe II: Strategien und Muster erfolgreichen Lehrerhandelns
}

\section{Johannes Mayr}

In einer Studie an Handelsakademien, in die 75 Lehrpersonen und deren Schülerinnen und Schülern einbezogen waren, wurde untersucht, welche Strategien der Klassenführung dazu beitragen, dass Schülerinnen und Schüler gut mitarbeiten und der Unterricht wenig durch Störungen belastet wird. Dabei stellten sich Merkmale der Unterrichtsgestaltung, der Förderung sozialer Beziehungen und der Verhaltenskontrolle als bedeutsam heraus. Das Verhalten erfolgreicher Lehrpersonen liegt innerhalb bestimmter, je nach Strategie unterschiedlich grosser Bandbreiten, wobei sich mehrere Muster erfolgreichen Führungsverhaltens unterscheiden lassen.

Es dürfte Konsens darüber bestehen, dass Lehrpersonen ihre Klassen so führen sollten, dass die Schülerinnen und Schüler sich im Unterricht intensiv dem Lernen widmen, dass die Lehr- und Lernprozesse möglichst wenig durch Störungen beeinträchtigt werden und dass mit den verbleibenden Konflikten konstruktiv umgegangen wird. Dies ist wichtig für das fachliche Lernen der Schülerinnen und Schüler, für ihr Wohlbefinden und nicht zuletzt auch für die berufliche $\mathrm{Zu}$ friedenheit von Lehrkräften (Weinert, 1996; Wendt, 2001).

Divergierend sind freilich die Vorstellungen darüber, wie diese Klassenführung beschaffen sein soll. Vergröbernd lassen sich drei Ansätze unterscheiden:

- Die angloamerikanischen Studien zum classroom management und die darauf basierenden Ratschläge an Lehrkräfte (Kounin, 1970; Emmer et al., 1985; Doyle, 1986) legen ein Führungsverhalten nahe, das durch ein präzises Regelsystem gekennzeichnet ist, welches das von der Lehrperson systematisch etabliert und hinsichtlich seiner Einhaltung genau überwacht wird.

- Vertreter eines personzentrierten Ansatzes sehen in der Gestaltung der LehrerSchüler-Beziehung den Angelpunkt für einen wirkungsvollen Umgang mit Problemverhalten (Gordon, 1997). Eine authentische, von Einfühlsamkeit und Wertschätzung getragene «Begegnung von Person zu Person» (Tausch \& Tausch, 1998) soll ein fachliches und persönliches «Lernen in Freiheit» (Rogers, 1969) ermöglichen. 
- Derzeit wird verstärkt die Gestaltung des Unterrichts als Mittel zur Steuerung des Schülerverhaltens gesehen (Helmke, 2004), wobei schon aus den Studien zum classroom management bekannte Faktoren wie Strukturiertheit und Reibungslosigkeit des Unterrichtsablaufs um Faktoren ergänzt werden, die anspruchsvolle kognitive Ziele und Formen selbstregulierten Lernens in den Blick nehmen (Schreiber, 1998).

Neben diesen, in den letzten Jahrzehnten unterschiedlich gewichteten Perspektiven (Jones \& Jones, 2004) tut sich bei der Analyse des Führungsverhaltens ein weiteres Spannungsfeld auf: Man kann das Hauptaugenmerk eher auf die Prävention von Unterrichtsstörungen und das Anregen von Lernprozessen richten (wie dies die Befunde der Unterrichtsforschung nahe legen) oder eher auf die Möglichkeiten wirkungsvoller Intervention, wenn Konflikte und Störungen den Unterrichtsfluss hemmen (wofür häufig Praktikerinnen und Praktiker Lösungen einfordern).

Der hier vorgestellten Studie liegt ein breiter, alle diese Sichtweisen integrierender Zugang zu Grunde. Ihre Basis bildet eine schon früher geleistete Sichtung der wissenschaftlichen Befunde zum oben aufgespannten Spektrum, eine Erkundung des einschlägigen Alltagswissens von Lehrpersonen und Schülerinnen und Schülern und dessen Verdichtung zu einer Sammlung pädagogischer Handlungsstrategien für Lehrkräfte (Mayr, Eder \& Fartacek, 1991). Diese Handlungsstrategien fanden ihren Niederschlag im «Linzer Diagnosebogen zur Klassenführung» (LDK), einem Fragebogen, der für Forschungszwecke ebenso geeignet sein sollte wie für die Selbstreflexion von Lehrpersonen und das Einholen von Feedback von Schulklassen.

In der hier vorgelegten Untersuchung sollen die Ergebnisse der bisher mit diesem Instrument durchgeführten Studien (insbesondere Mayr et al., 1991; Stockinger, 1999; Mayr, 2002, 2004; Eder, 2004) an sehr spezifischen Personengruppen - Lehrpersonen des Faches Betriebswirtschaftslehre (BWL) an Handelsakademien ${ }^{1}$ und deren Schüler - repliziert und ergänzt werden. Im Kern geht es dabei um folgende Fragestellungen:

(1) Wie hängen die im LDK erfassten Führungsstrategien mit unterschiedlichen Aspekten des Schülerverhaltens zusammen?

(2) Wie ist das pädagogische Handeln von Lehrpersonen beschaffen, die in der Klassenführung "erfolgreich» sind, und innerhalb welcher Bandbreite bewegt es sich?

(3) Lassen sich innerhalb dieser Bandbreite unterschiedliche Verhaltensmuster identifizieren?

Die Studie geht also einerseits von einem variablenorientierten Ansatz aus, der danach fragt, ob bzw. in welcher Weise einzelne potenziell relevante Lehrerverhaltensweisen mit dem Schülerverhalten korrelieren. Dabei wird entsprechend den obigen Ausführungen als Hypothese angenommen, dass ein Mehr an Nutzung der einzelnen Strategien im Allgemeinen mit einer besseren Mitarbeit der Schüler bzw. weniger Störverhalten einhergeht (vgl. Fragestellung 1). Die ange- 
strebte Identifizierung wirkungsvoller Handlungsmuster (Fragestellung 3) folgt dagegen einem typologischen Ansatz. Dieser beinhaltet die Vorstellung, dass es weniger die einzelnen Variablen des Lehrerverhaltens oder deren lineares Zusammenwirken sind, die den Erfolg ausmachen, sondern dass es unterschiedliche Merkmalskombinationen gibt, die in gleicher Weise zur Erreichung eines bestimmten Zielkriteriums (z.B. gute Mitarbeit der Schüler im Unterricht) beitragen. Hypothetisch wird davon ausgegangen, dass es sich dabei um ähnliche Verhaltensmuster handelt, wie sie sich in früheren Studien mit dem LDK gezeigt hatten (siehe dazu die Ausführungen im Ergebnisteil).

Da es sich um eine Korrelationsstudie handelt, muss die Frage nach der Wirkungsrichtung letztlich offen bleiben. Plausibel erscheint es jedoch - auch gestützt auf bisherige Studien zum LDK (insbesondere Stockinger, 1999) -, eine stärkere Wirkung des Lehrerverhaltens auf das Schülerverhalten anzunehmen. Aus Gründen der sprachlichen Vereinfachung ist im vorliegenden Text oft nur von dieser Wirkungsrichtung die Rede.

\section{Methode}

\section{Stichprobe und Datenerhebung}

Die Stichprobe besteht aus 75 BWL-Lehrinnen und -Lehrern aus 11 (von 17) oberösterreichischen Handelsakademien und je eine ihrer Klassen mit insgesamt 1619 Schülerinnen und Schüler. Es wurde danach getrachtet, möglichst jede Lehrperson, die dieses Fach an ihrer Schule vertritt, in die Erhebung einzubeziehen. Dieses Ziel wurde zwar nur an einigen Schulen erreicht, ein Vergleich der Daten der vollständig und der nur teilweise erfassten Schulen zeigt allerdings, dass die Stichprobe im Grossen und Ganzen als repräsentativ für die Lehrpersonen des Faches BWL in dieser Region bezeichnet werden kann. Das Alter der Lehrkräfte liegt zwischen 29 und 63 Jahren $(M=42,8)$, 55\% von ihnen sind weiblich. Die Schulstufen 9 bis 12 sind mit je 16 bis 18 Klassen praktisch gleich stark vertreten; für das Schuljahr 13 liegen nur Daten aus 7 Klassen vor, da diese Klassen aus technischen Gründen schwerer befragbar waren (z.B. weil das Schuljahr wegen der Reifeprüfung kürzer ist).

Die Daten wurden im Zuge einer umfangreicheren Studie zum Unterricht im Fach BWL von Studentinnen der Johannes Kepler Universität Linz im Rahmen ihrer Diplomarbeiten erhoben. ${ }^{2}$ Diese Studierenden sind die einzigen Personen, denen die Zuordnung der Daten zu den einzelnen Lehrkräften bekannt ist. Sie übernahmen auch die Rückmeldung der Individualergebnisse an die teilnehmenden Lehrpersonen.

Die folgenden Darstellungen konzentrieren sich auf die Angaben, die von den Schülerinnen und Schülern erfragt wurden. Derartige Daten dürften - speziell wenn man sie wie hier auf Klassenebene aggregiert - besonders aussagekräftig sein, u.a. weil sie auf Langzeitbeobachtungen basieren, Erfahrungen aus un- 
terschiedlichsten Situationen integrieren und durch Vergleiche zwischen verschiedenartigen Lehrpersonen abgesichert sind (Gruehn, 2000; Ditton, 2002). Die Sichtweise der Schülerinnen und Schüler ist auch insofern bedeutsam, als sie entscheidender als «objektive» Daten das Verhalten der Schüler im Unterricht beeinflusst und von Lehrpersonen als leicht zugängliche Reflexionshilfe für die Weiterentwicklung ihres pädagogischen Handelns genutzt werden kann (Mayr Eder \& Fartecek, 1991; Helmke, 2004).

\section{Die Erhebungsverfahren}

Die Lehrpersonen und die Schüler erhielten Fragebögen zum Lehrerverhalten, zum Schülerverhalten und zu thematisch einschlägigen Einstellungen vorgelegt, wobei die Items in der Lehrer- bzw. Schülerversion der Fragebögen inhaltlich identisch waren. Tabelle 1 enthält eine zusammenfassende Kurzbeschreibung der Schülerfragebögen.

Tabelle 1: Die wichtigsten Erhebungsverfahren

\begin{tabular}{|c|c|c|c|c|}
\hline Verfahren & Skalen & Beispielitems & $\begin{array}{l}\text { Anzahl } \\
\text { der } \\
\text { Items; } \\
\text { Alpha }\end{array}$ & $\begin{array}{l}\text { Antwort- } \\
\text { format }\end{array}$ \\
\hline \multirow{3}{*}{$\begin{array}{l}\text { Linzer Diagnosebogen } \\
\text { zur Klassenführung } \\
\text { (LDK; Mayr, Eder \& } \\
\text { Fartacek, 2005): aus- } \\
\text { gewählte Items aus der } \\
\text { Version für die Sekun- } \\
\text { darstufe II }\end{array}$} & $\begin{array}{l}\text { Unterrichts- } \\
\text { gestaltung }\end{array}$ & $\begin{array}{l}\text { Sie gliedert die Unterrichtsstunde } \\
\text { in Abschnitte, die gut } \\
\text { aufeinander passen. }\end{array}$ & $7 ; .89$ & \multirow[t]{3}{*}{$\begin{array}{l}\text { stimmt } \\
\text { nicht }(0) \\
\text { stimmt (4) }\end{array}$} \\
\hline & $\begin{array}{l}\text { Beziehungs- } \\
\text { förderung }\end{array}$ & Sie ist zu uns offen und ehrlich. & $7 ; .94$ & \\
\hline & $\begin{array}{l}\text { Verhaltens- } \\
\text { kontrolle }\end{array}$ & $\begin{array}{l}\text { Sie bemerkt alles, was in der } \\
\text { Klasse vor sich geht. }\end{array}$ & $7 ; .83$ & \\
\hline \multirow{4}{*}{$\begin{array}{l}\text { Fragebogen zum Pro- } \\
\text { blemverhalten von } \\
\text { Schülerinnen und } \\
\text { Schülern: } \\
\text { Weiterentwicklung des } \\
\text { Fragebogens von Farta- } \\
\text { cek, Eder \& Mayr } \\
\text { (1987) }\end{array}$} & Abschalten & $\begin{array}{l}\text { Im BWL-Unterricht bin ich mit } \\
\text { meinen Gedanken woanders. }\end{array}$ & $3 ; .90$ & \multirow{4}{*}{$\begin{array}{l}\text { fast nie }(0) \\
\text { manchmal (1) } \\
\text { oft (2) } \\
\text { fast immer (3) }\end{array}$} \\
\hline & Unruhe & $\begin{array}{l}\text {... unterhalte ich mich mit mei- } \\
\text { nem Sitznachbarn. }\end{array}$ & $3 ; .84$ & \\
\hline & Aggression & $\begin{array}{l}\text {... gerate ich in Streit mit Mit- } \\
\text { schülern. }\end{array}$ & $3 ; .74$ & \\
\hline & Regelverletzung & ... komme ich zu spät. & $3 ; .59$ & \\
\hline \multirow{3}{*}{$\begin{array}{l}\text { Fragebogen zu den } \\
\text { Lernstrategien im Unter- } \\
\text { richt / zu Hause: Adap- } \\
\text { tierung von Items aus } \\
\text { PISA } 2000 \text { (Kunter et } \\
\text { al., 2002) und dem Kie- } \\
\text { ler Lernstrategien-Inven- } \\
\text { tar (KLI; Baumert, Heyn } \\
\text { \& Köller, 1992) }\end{array}$} & $\begin{array}{l}\text { Einprägungs- } \\
\text { strategien }\end{array}$ & $\begin{array}{l}\text { Im BWL-Untericht } \\
\text { Beim Lernen zu Hause versuche } \\
\text { ich, mir alles Neue gut einzuprägen. }\end{array}$ & $\begin{array}{l}3 ; .83 \\
3 ; .63\end{array}$ & \multirow[t]{3}{*}{$\begin{array}{l}\text { fast nie }(0) \\
\text { manchmal (1) } \\
\text { oft (2) } \\
\text { fast immer (3) }\end{array}$} \\
\hline & $\begin{array}{l}\text { Elaborations- } \\
\text { strategien }\end{array}$ & $\begin{array}{l}\text {... überlege ich, wie der Stoff mit } \\
\text { dem zusammenhängt, was ich } \\
\text { schon gelernt habe. }\end{array}$ & $\begin{array}{l}3 ; .87 \\
3 ; .89\end{array}$ & \\
\hline & $\begin{array}{l}\text { Anstrengung } \\
\text { und Ausdauer }\end{array}$ & $\begin{array}{l}\text {... gebe ich nicht auf, auch wenn } \\
\text { der Stoff sehr schwierig ist. }\end{array}$ & $\begin{array}{l}3 ; .69 \\
3 ; .85\end{array}$ & \\
\hline \multirow{2}{*}{$\begin{array}{l}\text { Fragebogen zur Einstel- } \\
\text { lung der Schüler zur } \\
\text { Lehrperson und zum } \\
\text { Unterrichtsfach (Mayr, } \\
\text { Eder \& Fartacek, 1991) }\end{array}$} & $\begin{array}{l}\text { Einstellung zur } \\
\text { Lehrperson }\end{array}$ & Ich finde sie nett. & $3 ; .89$ & \multirow{2}{*}{$\begin{array}{l}\text { stimmt } \\
\text { nicht }(0) \\
\text { stimmt }(4)\end{array}$} \\
\hline & $\begin{array}{l}\text { Einstellung zum } \\
\text { Fach }\end{array}$ & Dieses Fach interessiert mich. & $3 ; .95$ & \\
\hline
\end{tabular}


Bezüglich des im Zentrum der Erhebung stehenden «Linzer Diagnosebogens zur Klassenführung» (LDK) ist zu ergänzen, dass dieser in einer erweiterten Version mit 31 Items zum Einsatz kam, um eine Reihe neuer Items auf ihre Brauchbarkeit zu testen. Für die hier vorgestellten Auswertungen wird jedoch nur auf die bereits in früheren Versionen erprobten 21 Items zurückgegriffen (diese sind bei Mayr, 2002, im Wortlaut wiedergegeben). Sie repräsentieren jeweils eine Facette («Strategie») des Führungsverhaltens. Die Ergebnisdarstellung nimmt einerseits auf diese 21 Facetten Bezug, andererseits auf deren Zusammenfassung zu drei Faktoren. Die Zuordnung der Facetten zu den Faktoren ist aus den Tabellen 2 und 3 ersichtlich. Sie wurde aus früheren Fragebogenversionen bzw. Studien übernommen, um den Vergleich mit diesen zu erleichtern. Neuberechnungen bestätigen die Faktorenstruktur für die gegenständliche Stichprobe, würden jedoch eine teilweise andere Itemzuordnung nahe legen. Die hohen Reliabilitätskoeffizienten auch in der aktuellen Stichprobe (siehe Tabelle 1) legitimieren jedoch die Verwendung der Original-Skalen (die durch Skalenanalysen an umfangreichen Stichproben mit unterschiedlicher Zusammensetzung abgesichert sind; Mayr, Eder \& Fartacek, 2005).

\section{Ergebnisse}

\section{Zur übereinstimmung von Lehrer- und Schülersicht}

Sämtliche im Folgenden dargestellte Ergebnisse basieren - soweit es um Einschätzungen des Lehrer- und Schülerverhaltens geht - auf den Angaben der Schülerinnen und Schüler, die auf Klassenebene aggregiert wurden $(\mathrm{N}=75)$. Da diese Variablen jedoch auch aus Sicht der Lehrkräfte erhoben wurden, soll hier kurz auf die Übereinstimmung dieser Angaben Bezug genommen werden (eine ausführlichere Darstellung wird an anderem Ort erfolgen).

Korrelationsstatistisch betrachtet liegt die Übereinstimmung bezüglich der Faktoren des Lehrerverhaltens (Unterrichtsgestaltung, Beziehungsförderung, Verhaltenskontrolle) zwischen .249 und .345 , bezüglich des gut beobachtbaren Störverhaltens der Schüler (Unruhe, Aggression und Regelverletzung) zwischen .374 und .560 und sie beträgt bezüglich des schlechter beobachtbaren «Abschaltens» der Schüler im Unterricht (nicht signifikante) .143. Bezüglich der Lernstrategien erreicht keine der Korrelationen das übliche Signifikanzniveau.

Die Übereinstimmung ist also - wie bei vergleichbaren Untersuchungen nicht allzu hoch. Bezüglich des Lehrerverhaltens vergrössert sich die Diskrepanz der Sichtweisen noch dadurch, dass ein deutlicher Mittelwertsunterschied auftritt: Die Lehrpersonen schätzen ihr Verhalten bezüglich aller drei Faktoren um mehr als eine Standardabweichung günstiger (im Sinne von: verstärkter Anwendung der betreffenden Strategien) ein. Bezüglich der Einschätzung des Schülerverhaltens zeigen sich keine einheitlichen Trends. 


\section{Korrelative Beziehungen zwischen Lehrer- und Schülerverhalten}

Die Auswahl der Handlungsstrategien war von der Vorstellung geleitet gewesen, dass sie einen Beitrag zu hohem Lernengagement der Schüler leisten bzw. störungspräventiv wirken sollten. Daraus und aus entsprechenden, diese Annahme theoretisch fundierenden Konzepten und empirisch absichernden Studien ergibt sich, dass den Korrelationsberechnungen im Allgemeinen gerichtete Hypothesen zu Grunde liegen. Eine Ausnahme davon bildet die Strategie «Bestrafung unerwünschten Verhaltens», für die sich wiederholt Anhaltspunkte fanden, dass sie zumindest für einzelne Zielkriterien kontraproduktiv wirkt (siehe Mayr, Eder \& Fartecek, 1991; Mayr, 2002, 2004). Zwecks Vereinfachung der Darstellung wird im Folgenden generell eine zweiseitige Hypothesenprüfung durchgeführt, was ein wenig auch der Gefahr entgegenwirken soll, Scheinzusammenhänge zu präsentieren (siehe zu diesem Problem Rost, 2005).

Tabelle 2: Zusammenhänge zwischen den Faktoren (I bis III) bzw. den ihnen zugeordneten Facetten der Klassenführung (1-21) und dem Problemverhalten der Schüler

\begin{tabular}{|l|c|c|c|c|}
\hline & Abschalten & Unruhe & Aggression & $\begin{array}{c}\text { Regel- } \\
\text { verletzung }\end{array}$ \\
\hline FAKTOR I: Unterrichtsgestaltung & $-.498^{* * *}$ & $-.371^{* * *}$ & $-.510^{* * *}$ & $-.251^{*}$ \\
\hline 1. bedeutsame Lernziele & $-.584^{* * *}$ & $-.402^{* * *}$ & $-.383^{* * *}$ & $-.316^{* *}$ \\
\hline 2. strukturierter Unterricht & $-.439^{* * *}$ & $-.250^{*}$ & $-.428^{* * *}$ & -.203 \\
\hline 3. klare Arbeitsanweisungen & $-.243^{*}$ & $-.264^{*}$ & $-.373^{* * *}$ & -.145 \\
\hline 4. interessanter Unterricht & $-.655^{* * *}$ & $-.493^{* * *}$ & $-.394^{* * *}$ & $-.272^{*}$ \\
\hline 5. Fachkompetenz & $-.375^{* * *}$ & $-.287^{*}$ & $-.334^{* *}$ & -.107 \\
\hline 6. positive Erwartungshaltung & $-.280^{*}$ & -.213 & $-.459^{* * *}$ & -.218 \\
\hline 7. Verlässlichkeit & -.169 & -.134 & $-.416^{* * *}$ & -.118 \\
\hline FAKTOR II: Beziehungsförderung & $-.337^{* *}$ & $-.304^{* *}$ & $-.458^{* * *}$ & -.191 \\
\hline 8. Wertschätzung & $-.272^{*}$ & $-.326^{* *}$ & $-.556^{* * *}$ & $-.235^{*}$ \\
\hline 9. Verstehen & -.191 & -.168 & $-.437^{* * *}$ & -.106 \\
\hline 10. Authentizität & $-.370^{* * *}$ & $-.434^{* * *}$ & $-.481^{* * *}$ & $-.261^{*}$ \\
\hline 11. Humor & $-.244^{*}$ & -.205 & $-.432^{* * *}$ & -.169 \\
\hline 12. Kommunikation & $-.318^{* *}$ & $-.293^{*}$ & -.227 & -.088 \\
\hline 13. Schülermitbestimmung & $-.320^{* *}$ & -.213 & $-.358^{* *}$ & -.138 \\
\hline 14. Gemeinschaftsförderung & $-.335^{* *}$ & -.220 & $-.312^{* *}$ & -.178 \\
\hline FAKTOR III: Verhaltenskontrolle & $-.290^{*}$ & $-.240^{*}$ & -.005 & -.072 \\
\hline 15. klare Verhaltenserwartungen & $-.272^{*}$ & $-.391^{* * *}$ & $-.268^{*}$ & -.158 \\
\hline 16. Beschäftigung der Schüler & -.153 & -.105 & -.091 & -.100 \\
\hline 17. Kontrolle der Lernarbeit & -.088 & .123 & .088 & .107 \\
\hline 18. „Allgegenwärtigkeit“ & $-.286^{*}$ & $-.292^{*}$ & -.109 & -.113 \\
\hline 19. Verstärkung erwünschten Verh. & $-.357^{* *}$ & $-.312^{* *}$ & -.162 & -.139 \\
\hline 20. rasches Eingreifen bei Störung & -.200 & $-.234^{*}$ & .108 & -.127 \\
\hline 21. Bestrafung unerwünschten Verh. & -.183 & -.145 & .218 & .062 \\
\hline
\end{tabular}

$\mathrm{N}=75$ Lehrpersonen bzw. Klassen; Produktmoment-Korrelationen, ${ }^{*} \mathrm{p}<.05,{ }^{* *} \mathrm{p}<.01,{ }^{* * *} \mathrm{p}<.001$ (zweiseitig) 
Tabelle 2 stellt die Zusammenhänge dar, die sich in der Gesamtstichprobe zwischen dem Führungsverhalten der Lehrkräfte und dem Mitarbeits-bzw. Störverhalten ihrer Klassen zeigen. Die Ergebnisse sprechen zumindest bezüglich der Faktoren Unterrichtsgestaltung und Beziehungsförderung für die Gültigkeit der postulierten Hypothesen. Die Korrelationen sind teilweise recht deutlich ausgefallen und sie sind im Allgemeinen signifikant bzw. weisen zumindest ein Vorzeichen in die erwartete Richtung auf. Beim Faktor Verhaltenskontrolle zeigen sich - ebenfalls übereinstimmend mit den bisherigen Befunden - differenzielle Effekte: Insbesondere bei den Strategien «Kontrolle der Lernarbeit» und «Bestrafung unerwünschten Verhaltens» deuten sich Zusammenhänge an, die in Richtung ungünstiger (jedenfalls nicht in Richtung wünschenswerter) Effekte interpretiert werden können. Allerdings muss - da es sich um eine Korrelationsstudie handelt - auch die umgekehrte Kausalrichtung mitbedacht werden: In Problemklassen könnten sich Lehrpersonen eher genötigt sehen, disziplinierend einzugreifen.

Einzelne Faktoren (z.B. die Unterrichtsgestaltung) und Facetten des Lehrerverhaltens (z.B. die Strategie, interessant zu unterrichten) klären auch für sich allein genommen zwischen 25 und 40 Prozent der Varianz des Schülerverhaltens auf. Zu diesem Ergebnis gelangt man, wenn man aus den in Tabelle 2 angeführten Korrelationen die entsprechenden Determinationskoeffizenten $\left(\mathrm{r}^{2}\right)$ berechnet. Wenn man mittels (schrittweiser) Regression auf das Erklärungspotenzial aller prognosetauglichen Facetten zurückgreift, dann ergeben sich entsprechend höhere Varianzaufklärungen $\left(\mathrm{R}^{2}\right)$ : bezüglich des Kriteriums "Abschalten» sind es $56 \%$, bezüglich «Unruhe» 38\%, bezüglich «Aggression» 41\% und bezüglich «Regelverletzung» 14\%. Bei Werten in dieser Höhe ist neben der statistischen Signifikanz zweifelsohne auch praktische Relevanz gegeben (Bortz \& Döring, 2003).

Die Realisierung der angeführten Faktoren bzw. Facetten korreliert jedoch nicht nur mit dem Mitarbeits- bzw. Störverhalten. Es zeigen sich auch (nicht in der Tabelle dargestellte) relativ enge Beziehungen zu den im Unterricht von den Schülerinnen und Schülern angewendeten Lernstrategien. Dabei ist es vor allem die Unterrichtsgestaltung, die durchschlägt: Sie korreliert mit den Einprägungsstrategien zu .534, mit den Elaborationsstrategien zu .366 und mit der Anstrengung zu .579. Der Faktor Unterrichtsgestaltung korreliert auch mit den im häuslichen Lernen angewandeten Strategien mit bis zu .413. Das Fördern sozialer Beziehungen und die Kontrolle des Schülerverhaltens weisen zu diesen Kriterien vergleichsweise schwächere Beziehungen auf.

Interessant erscheinen auch die (ebenfalls nicht tabellarisch dargestellten) $\mathrm{Zu}$ sammenhänge des Führungsverhaltens mit den Einstellungen der Schüler zur Lehrkraft und zum Unterrichtsfach: Sie betragen beim Faktor Unterrichtsgestaltung .901 bzw. .552, beim Faktor Beziehungsförderung .842 bzw. .449 und beim Faktor Verhaltenskontrolle .332 bzw. .376. Die Korrelationen sind jeweils sehr signifikant und ihrer Grössenordnung nach durchaus relevant. 


\section{Position und Bandbreite erfolgreichen Lehrerverhaltens}

Ein neben der Berechnung von korrelativen Beziehungen weiterer Weg, potenziell wirkungsvolle Strategien der Klassenführung zu identifizieren, besteht darin, das Verhalten erfolgreicher Lehrerinnen und Lehrer mit dem weniger erfolgreicher Lehrkräfte zu kontrastieren. Dabei können die Mittelwerte des Verhaltens der beiden Gruppen verglichen werden bzw. es kann die Spannweite zwischen Minimal- und Maximalwert («Bandbreite») ermittelt werden, innerhalb derer das Handeln der erfolgreichen Gruppe angesiedelt ist. Vom Mittelwertsvergleich sind dabei im Wesentlichen dieselben Erkenntnisse zu erwarten wie von der bereits erörterten Berechnung von Korrelationen zwischen Lehrer- und Schülerverhalten, die Analyse der Bandbreite erlaubt dagegen Rückschlüsse auf den Spielraum, der bei den einzelnen Strategien besteht. Den bisherigen Erhebungen mit dem LDK zufolge ist dieser stark von der Strategie abhängig: Relativ wenig Spielraum scheint es vor allem bei Strategien aus den Bereichen Unterrichtsgestaltung und Verhaltenskontrolle zu geben, relativ viel dagegen beim Ausmass an Beziehungsförderung (Mayr, Eder \& Fartecek, 1991; Mayr, 2002).

Um diesen Sachverhalt bei der vorliegenden Stichprobe zu untersuchen, wurde diese nach dem Ausmass, in dem in den einzelnen Klassen nach Angabe der Schüler «Abschalten» und «Unruhe» auftrat in zwei Gruppen geteilt: Wenn der Summenwert aus diesen beiden Indikatoren für (mangelnde) Mitarbeit unter dem Median lag, wurde die betreffende Lehrperson der Gruppe der «erfolgreiche(re)n» Lehrkräfte $(\mathrm{N}=37)$ zugewiesen. Die übrigen Lehrerinnen und Lehrer $(\mathrm{N}=38)$ bilden die Gruppe der «weniger Erfolgreichen». (Eine an sich wünschenswerte deutlichere Trennung der zu vergleichenden Gruppen - z.B. durch Beschränkung auf das obere und das untere Quartil - erschien auf Grund des Stichprobenumfangs nicht zielführend.)

Tabelle 3 ermöglicht einen Vergleich der Mittelwerte und der Bandbreite des Verhaltens dieser beiden Gruppen. Die Mittelwerte der erfolgreicheren Lehrergruppe liegen - in Übereinstimmung mit den Erwartungen und den oben vorgestellten Ergebnissen - zumeist signifikant über der Vergleichsgruppe. Die praktische Bedeutsamkeit der Mittelwertsdifferenzen ist mit Effektstärken bis 1,22 (bei der Facette «interessanter Unterricht») bei vielen Strategien durchaus beachtlich. Andererseits ist die Bandbreite gemessen an der theoretisch möglichen Spannweite von fünf Skalenstufen oft ausgesprochen gross: Bei der Facette Kommunikation (Item-Text: "Wir reden mit ihr auch über den Unterricht und unsere Klasse») erreicht sie sogar einen Wert von 3,45 Stufen. Vergleichsweise gering fällt sie bei Fachkompetenz und Verstärkung erwünschten Verhaltens mit 1,47 bzw. 1,38 Stufen aus. Interessanterweise sind sowohl die Mittelwerte als auch die Bandbreite in der vorliegenden Stichprobe erfolgreicher BWL-Lehrer weitgehend gleich mit einer Stichprobe von Lehrpersonen aus allen Fächern derselben Schulart, die rückblickend von Absolventen der Handelsakademie als "gute Lehrkräfte» nominiert worden waren (Mayr, 2002). 
Tabelle 3: Kennwerte der Faktoren (I bis III) bzw. der ibnen zugeordneten Facetten der Klassenführung (1 bis 21) bei unterschiedlich erfolgreichen Lehrpersonen

\begin{tabular}{|c|c|c|c|c|c|c|c|c|c|c|c|}
\hline & \multicolumn{4}{|c|}{$\begin{array}{l}\text { Weniger erfolgreiche } \\
\text { Lehrperson }\end{array}$} & \multicolumn{4}{|c|}{$\begin{array}{l}\text { Erfolgreiche } \\
\text { Lehrperson }\end{array}$} & \multicolumn{3}{|c|}{$\begin{array}{l}\text { Gruppen- } \\
\text { vergleich }\end{array}$} \\
\hline & Min & Max & M & SD & Min & Max & M & SD & $\mathrm{t}$ & $\mathrm{d}$ & $\mathrm{p}$ \\
\hline FAKTOR I: Unterrichtsgestaltung & 1.25 & 3.52 & 2.46 & .46 & 1.70 & 3.68 & 2.93 & .45 & 4.46 & 1.03 & *** \\
\hline 1. bedeutsame Lernziele & 1.64 & 3.46 & 2.81 & .47 & 2.07 & 3.87 & 3.26 & .43 & 4.32 & 1.00 & *** \\
\hline 2. strukturierter Unterricht & .45 & 2.96 & 1.89 & .53 & .63 & 3.82 & 2.41 & .64 & 3.78 & .88 & *** \\
\hline 3. klare Arbeitsanweisungen & 1.05 & 3.54 & 2.48 & .57 & .93 & 3.86 & 2.95 & .54 & 3.69 & .85 & $* * *$ \\
\hline 4. interessanter Unterricht & .38 & 3.64 & 1.80 & .67 & .87 & 3.65 & 2.60 & .64 & 5.30 & 1.22 & *** \\
\hline 5. Fachkompetenz & 1.19 & 4.00 & 3.16 & .72 & 2.53 & 4.00 & 3.60 & .38 & 3.36 & .76 & *** \\
\hline 6. positive Erwartungshaltung & .94 & 3.80 & 2.39 & .74 & 1.04 & 3.91 & 2.65 & .80 & 1.48 & .34 & \\
\hline 7. Verlässlichkeit & 1.07 & 3.57 & 2.70 & .59 & 1.22 & 4.00 & 3.03 & .66 & 2.33 & .53 & * \\
\hline FAKTOR II: Beziehungsförderung & .85 & 3.28 & 2.21 & .57 & 1.27 & 3.48 & 2.55 & .56 & 2.54 & .60 & * \\
\hline 8. Wertschätzung & .32 & 3.77 & 2.65 & .76 & 1.09 & 3.89 & 2.98 & .67 & 2.02 & .46 & * \\
\hline 9. Verstehen & .55 & 3.29 & 2.04 & .64 & 1.20 & 3.33 & 2.26 & .66 & 1.41 & .34 & \\
\hline 10. Authentizität & 1.56 & 3.57 & 2.75 & .53 & 2.08 & 3.87 & 3.15 & .43 & 3.60 & .83 & $* * *$ \\
\hline 11. Humor & .62 & 3.79 & 2.46 & .75 & 1.04 & 3.96 & 2.71 & .76 & 1.47 & .33 & \\
\hline 12. Kommunikation & .81 & 3.90 & 1.88 & .76 & .24 & 3.69 & 2.32 & .83 & 2.41 & .55 & * \\
\hline 13. Schülermitbestimmung & 1.23 & 3.00 & 2.06 & .44 & 1.36 & 3.60 & 2.29 & .49 & 2.17 & .49 & * \\
\hline 14. Gemeinschaftsförderung & .41 & 3.57 & 1.66 & .72 & .73 & 3.81 & 2.10 & .82 & 2.49 & .57 & * \\
\hline FAKTOR III: Verhaltenskontrolle & 1.50 & 3.15 & 2.37 & .41 & 1.50 & 3.23 & 2.65 & .36 & 3.13 & .73 & ** \\
\hline 15. klare Verhaltenserwartungen & 1.75 & 3.64 & 2.79 & .42 & 2.00 & 3.81 & 3.12 & .35 & 3.75 & .85 & *** \\
\hline 16. Beschäftigung der Schüler & 1.50 & 3.50 & 2.63 & .54 & 1.33 & 3.78 & 2.88 & .55 & 1.93 & .46 & \\
\hline 17. Kontrolle der Lernarbeit & .73 & 3.53 & 2.29 & .71 & .67 & 3.35 & 2.47 & .65 & 1.10 & .26 & \\
\hline 18. „Allgegenwärtigkeit“ & .85 & 2.95 & 1.68 & .53 & .67 & 3.04 & 2.07 & .58 & 3.03 & .70 & ** \\
\hline 19. Verstärkung erwünschten Verh. & 1.24 & 3.43 & 2.56 & .49 & 2.09 & 3.47 & 2.78 & .35 & 2.21 & .52 & * \\
\hline 20. rasches Eingreifen bei Störung & 1.03 & 3.75 & 2.61 & .66 & 1.93 & 3.84 & 2.96 & .53 & 2.54 & .58 & * \\
\hline 21. Bestrafung unerwünschten Verh. & .34 & 3.40 & 2.00 & .74 & .95 & 3.58 & 2.27 & .68 & 1.64 & .38 & \\
\hline
\end{tabular}

$\mathrm{N}=75$ (38 weniger erfolgreiche und 37 erfolgreiche Lehrpersonen)

M Mittelwert, SD Standardabweichung, t Prüfgrösse im Signifikanztest, d Effektstärke $\mathrm{t}$-Tests, $\mathrm{df}=73,{ }^{*}<\mathrm{p} .05,{ }^{* *} \mathrm{p}<.01,{ }^{* * *} \mathrm{p}<.001$ (zweiseitig) 
Wenn man ergänzend die Bandbreite des Verhaltens bei den weniger erfolgreichen Lehrpersonen in die Betrachtung mit einbezieht, dann fallen die grossen Überlappungen zwischen diesen beiden Gruppen auf. Das mag zum Teil daran liegen, dass sie durch Medianhalbierung gebildet wurden und daher ein grosser Teil von Personen beider Gruppen in ihrer Wirksamkeit (und auch im dahinter stehenden Verhalten) im Durchschnittsbereich angesiedelt ist und mehr oder weniger zufällig in eine der beiden Vergleichsgruppen geriet. Dennoch unterstreicht diese Überlappung, dass es keine klare Trennlinie zwischen unterschiedlich erfolgreichen Lehrkräften gibt.

\section{Muster erfolgreichen Führungsverhaltens}

Schon Alltagsbeobachtungen legen nahe, dass es nicht eine spezielle Art erfolgreichen pädagogischen Handelns gibt, sondern dass es vermutlich unterschiedliche, durchaus zweckmässige «Wege der Klassenführung» geben dürfte. In früheren Studien ( Mayr Eder \& Fartecek, 1991; Mayr, 2004) hatten sich vier solcher Wege bzw. Handlungsmuster herauskristallisiert, die durch jeweils unterschiedliche Merkmalskombinationen gekennzeichnet sind. Manche Lehrerinnen und Lehrer scheinen in dieser Hinsicht einen persönlichen Stil aufzuweisen, den sie relativ unabhängig von äusseren Umständen realisieren (dann könnte man auch von einem «Lehrertyp» sprechen), andere beschreiten je nach Situation unterschiedliche Wege.

Tabelle 4: Muster des Führungsverhaltens bei erfolgreichen Lehrpersonen

\begin{tabular}{|c|c|c|c|c|c|c|c|}
\hline Faktor & $\begin{array}{l}\text { Muster A } \\
\text { M (SD) }\end{array}$ & $\begin{array}{l}\text { Muster B } \\
\text { M (SD) }\end{array}$ & $\begin{array}{c}\text { Muster C } \\
\text { M (SD) }\end{array}$ & $\begin{array}{l}\text { Muster D } \\
\text { M (SD) }\end{array}$ & $\begin{array}{l}\mathrm{F} \\
\mathrm{p}\end{array}$ & $\mathrm{f}$ & $\begin{array}{l}\text { Gruppen- } \\
\text { vergleich }\end{array}$ \\
\hline $\begin{array}{l}\text { Unterrichtsgestal- } \\
\text { tung }\end{array}$ & $\begin{array}{l}3.34 \\
(.16)\end{array}$ & $\begin{array}{l}3.25 \\
(.22)\end{array}$ & $\begin{array}{l}2.77 \\
(.20)\end{array}$ & $\begin{array}{l}2.32 \\
(.41)\end{array}$ & $\begin{array}{c}28.94 \\
* * *\end{array}$ & .84 & $\mathrm{~A}, \mathrm{~B}>\mathrm{C}>\mathrm{D}$ \\
\hline $\begin{array}{l}\text { Beziehungsförde- } \\
\text { rung }\end{array}$ & $\begin{array}{l}2.81 \\
(.38)\end{array}$ & $\begin{array}{l}3.23 \\
(.17)\end{array}$ & $\begin{array}{l}2.38 \\
(.26)\end{array}$ & $\begin{array}{l}1.78 \\
(.28)\end{array}$ & $\begin{array}{l}33.40 \\
* * *\end{array}$ & .86 & $\mathrm{~B}>\mathrm{A}>\mathrm{C}>\mathrm{D}$ \\
\hline Verhaltenskontrolle & $\begin{array}{l}3.03 \\
(.14)\end{array}$ & $\begin{array}{l}2.40 \\
(.14)\end{array}$ & $\begin{array}{l}2.51 \\
(.22)\end{array}$ & $\begin{array}{l}2.61 \\
(.53)\end{array}$ & $\begin{array}{c}9.07 \\
* * *\end{array}$ & .66 & $A>D, C, B$ \\
\hline $\mathrm{N}$ & 10 & 7 & 13 & 7 & & & \\
\hline
\end{tabular}

$\mathrm{N}=37$; M Mittelwert, SD Standardabweichung,

einfache Varianzanalysen $\left({ }^{* * *} \mathrm{p}<.001\right)$ mit anschliessendem Vergleich der Gruppenmittelwerte mittels Sheffé-Tests (Signifikanzniveau generell .05); f = Effektstärke

Anhand der vorliegenden Daten wurde ebenfalls versucht, mittels einer hierarchischen Clusteranalyse solche Muster zu identifizieren, und zwar anhand der auf Faktorenebene zusammengefassten Angaben zum Führungsverhalten der Teilstichprobe der 37 «erfolgreich(ere)n Lehrpersonen». Nach dem Kriterium des Fehlervarianz-Anstiegs fiel die Entscheidung zu Gunsten einer vier Gruppen umfassenden Klassifikation, deren Charakteristika in anschliessenden Varianza- 
nalysen ermittelt wurden (für Details siehe Tabelle 4). Die Muster werden im Folgenden kurz charakterisiert und mit den bisherigen Befunden und MusterBezeichnungen (in der Fassung von Mayr, 2004) in Beziehung gesetzt:

- Muster $A$ ist vor allem durch einen hohen Wert bei der Unterrichtsgestaltung und eine ausgeprägte Tendenz zur Kontrolle des Schülerverhaltens gekennzeichnet, weist aber auch einen passablen Score im Fördern sozialer Beziehungen auf. Es entspricht im Wesentlichen dem in früheren Untersuchungen gefundenen fachorientiertem Muster, die Werte beim Kontrollverhalten liegen allerdings höher.

- Muster B liegt bezüglich der Unterrichtsgestaltung praktisch mit A gleich auf, es wird jedoch versucht, das Verhalten der Schüler bevorzugt durch Beziehungsförderung zu beeinflussen. Bezüglich der Verhaltenskontrolle ist der Wert der niedrigste von allen Gruppen. Dieses Muster deckt sich im Kern mit dem kommunikativ-beziehungsorientierten Handlungsmuster.

- Muster $C$ ist durch ein ausgeglichenes, überall im Mittelbereich liegendes Verhalten charakterisiert. Ein ähnlich neutrales Muster hat sich - auf unterschiedlichem Niveau - auch in den früheren Studien gezeigt.

- Muster D liegt bezüglich Unterrichtsgestaltung und Beziehungsförderung signifikant unter allen anderen Gruppen. Dass es dennoch letztlich eine erfolgreiche Klassenführung ermöglicht, liegt vermutlich an der ausreichend praktizierten Verhaltenskontrolle. Es weist in seinem Profil grosse Ähnlichkeit mit dem disziplinierenden Handlungsmuster aus den bisherigen Studien auf.

Bezogen auf das Merkmal «Mitarbeit» unterscheiden sich die vier Muster nicht (es war dies ja auch das Kriterium für die Klassifikation als «erfolgreiche» Lehrpersonen). Auch hinsichtlich der anderen Schülerverhaltensweisen deuten sich nur geringe Unterschiede an, lediglich die Muster A und D weichen bezüglich aggressiven Verhaltens signifikant voneinander ab. Hier dürften die typenspezifischen Differenzen bei der Unterrichtsgestaltung und beim Fördern sozialer Beziehungen durchschlagen (vgl. dazu die Korrelationsmuster dieser Variablen in Tabelle 2). Es finden sich auch Hinweise auf Differenzen bezüglich der Strategien, nach denen ausserhalb des Unterrichts für das Fach BWL gelernt wird: Beim (kontrollierenden) Muster D sind die Einprägungsstrategien signifikant verbreiteter als bei den anderen Gruppen, beim (beziehungsfördernden) Muster $\mathrm{B}$ sind sie das am wenigsten. Spiegelbildlich dazu besetzen diese beiden Muster die Randpositionen bei den Elaborationsstrategien.

Die Einstellung der Schüler zu den Lehrkräften ist deutlich vom Handlungsmuster abhängig: Lehrpersonen mit den Mustern A und B werden mit Einstellungs-Mittelwerten von 3.28 bzw. 3.21 signifikant gegenüber den anderen bevorzugt, aber auch zu C (2.66) und D (1.97) sind die Schüler noch positiv bzw. zumindest neutral eingestellt. Ein Einfluss des Handlungsmusters auf die Einstellung zum Fach ist statistisch nicht nachweisbar.

Das Handlungsmuster A wird bevorzugt in den niedrigeren Klassen angewandt, Muster B in den höheren - insbesondere in der Abschlussklassen. Die 
Klassengrösse und die Leistungsfähigkeit der Klasse (definiert über den Notenschnitt aus den Hauptfächern) spielen keine Rolle für das Handlungsmuster, ebenso nicht das Geschlecht der Lehrperson, ihr Alter und ihre Berufserfahrung.

\section{Zusammenfassung und Diskussion}

Die Ergebnisse der Studie belegen einmal mehr die enge Verknüpfung von Lehrer- und Schülerverhalten. Auf Grund des Untersuchungsdesigns kann zwar keine Aussage bezüglich der Wirkungsrichtung gemacht werden, in Zusammenschau mit anderen Forschungen und angesichts der Tatsache einer hierarchischen Beziehung zwischen den Lehrpersonen und ihren Schülerinnen und Schülern erscheinen jedoch folgende Zusammenfassungen und Interpretationen der Ergebnisse zulässig, die von deutlichen Einflüssen des Lehrerhandelns ausgehen:

- Merkmale der Unterrichtsgestaltung, insbesondere die Interessantheit des Unterrichts, seine klare Strukturierung und eine für die Schüler erkennbare Bedeutsamkeit der Lehrinhalte für ihr späteres Leben korrelieren substanziell mit dem Engagement der Schüler im Unterricht und trennen tendenziell zwischen erfolgreichen Lehrpersonen und ihren in der Klassenführung weniger wirkungsvollen Kolleginnen und Kollegen.

- Dasselbe gilt für Strategien, die auf eine Förderung sozialer Beziehungen innerhalb der Klasse zielen, dazu zählen insbesondere Authentizität der Lehrperson und ein wertschätzender Umgang mit den Schülern. Zusammen mit einfühlsamem Verstehen und einer ausgeglichen-humorvollen Haltung sind dies Handlungsweisen, die auch präventiv gegen Aggressionen in der Klasse wirken.

- Massnahmen der Verhaltenskontrolle, die sich in Studien bei jüngeren Schülerinnen und Schülern immer wieder als besonders effizient herausgestellt haben, dürften bei den (älteren) Schülern der vorliegenden Stichprobe weniger wirksam zu sein (vgl. ähnliche Befunde bei Eder, 2004). Klare Verhaltenserwartungen, Aufmerksamkeit für die Vorgänge im Klassenzimmer und Anerkennen konstruktiver Verhaltensweisen der Schüler scheinen aber auch bei ihnen günstige Effekte zu haben.

Wenn Lehrpersonen die genannten Handlungsweisen in höherem Ausmass realisieren, dann treten im Schnitt nicht nur weniger Unterrichtsstörungen auf, ihre Schülerinnen und Schüler sind $z u$ diesen Lehrpersonen auch deutlich positiver eingestellt als zu Lehrkräften mit weniger wirkungsvoller Klassenführung (vgl. identische Ergebnisse bei Mayr Eder \& Fartecek, 1991; Stockinger, 1999; Mayr, 2004).

Diese Befunde legen den Gedanken nahe, Lehrerinnen und Lehrer sollten ihr Handeln an ihnen orientieren. Überlegungen in diese Richtung sind zwar grundsätzlich zielführend, sie sind jedoch vor dem Hintergrund der folgenden Ergebnisse zu relativieren: 
- Das Verhalten der erfolgreichen Lehrpersonen variiert innerhalb einer je nach Handlungsstrategie unterschiedlich grossen Bandbreite. Das weist auf den Spielraum hin, der für individuelle Präferenzen der Lehrkraft verbleibt: Kontaktbereite, sich gern auf die Erlebniswelt anderer einlassende Lehrpersonen können z.B. ihre Klassen durchaus kommunikativer führen als gegenteilig disponierte Lehrerinnen und Lehrer (vgl. Befunde über entsprechende Zusammenhänge bei Mayr \& Neuweg, 2006). Die Bandbreite erlaubt auch ein flexibles Eingehen auf situative Bedingungen: Möglicherweise ist es z.B. zweckmässig, in bestimmten Klassen eine verstärkte Verhaltenskontrolle auszuüben oder bei einem bestimmten Lehrstoff strukturierter vorzugehen und bei einem anderen offenere Lernformen vorzusehen. Dass Lehrpersonen diesen Spielraum funktional nützen, belegen Ergebnisse aus anderen Studien (z.B. Mayr Eder \& Fartecek, 1991; Gruehn, 2000).

- Als Ausdruck dieses Spielraums können auch die unterschiedlichen Verhaltensmuster interpretiert werden, die sich unter den erfolgreichen Lehrpersonen der Stichprobe identifizieren liessen. In ihnen manifestieren sich die erwähnten individuellen und situativen Rahmenbedingungen des Handelns, sie lassen aber auch vermuten, dass eventuelle Defizite in einem Bereich des Lehrerverhaltens zumindest teilweise durch Stärken in anderen Bereichen wettgemacht werden können, z.B. dass sich eine geringere Bereitschaft oder Kompetenz zur Verhaltenskontrolle durch verstärktes Bemühen um die Förderung sozialer Beziehungen ausgleichen lässt (vgl. ähnliche Befunde und Überlegungen zur Kompensierbarkeit bei Weinert \& Helmke, 1996).

Ein zwar nicht überraschendes aber dennoch bedenkenswertes Ergebnis ist die geringe Übereinstimmung zwischen der Selbsteinschätzung des eigenen pädagogischen Handelns durch Lehrpersonen und der Fremdeinschätzung durch ihre Schüler. Lehrerinnen und Lehrern, die ihre Führungsstrategien klären und weiterentwickeln möchten, wäre demnach zu empfehlen, auch Schülerrückmeldungen einzuholen, um ihr Bild abzurunden. Dafür und für die Selbsteinschätzung kann der in der Studie verwendete «Linzer Diagnosebogen zur Klassenführung» (LDK) verwendet werden. Er ist in verschiedenen Versionen (z.B. für unterschiedlichen Schulstufen) samt entsprechenden Vergleichswerten, statistischen Kennzahlen und Hinweisen für seinen Einsatz als Download verfügbar (http://ius.uni-klu.ac.at/projekte/ldk/). Es gibt auch mehrere für Praktiker verfasste einschlägige Publikationen, wobei jene von Lohmann (2005) besonders ausführlich auf den LDK Bezug nimmt, ihn mit anderen Verfahren verbindet und methodische Anregungen zur Nutzung dieser Materialien bietet.

\section{Anmerkungen}

1 Die Handelsakademien zählen in Österreich zu den «berufsbildenden höheren Schulen». Sie führen Absolventinnen und Absolventen der Sekundarstufe I (Hauptschulen oder Unterstufen des Gymnasiums) in einer fünfjährigen Ausbildung zur Reifeprüfung, wobei sie 
neben Allgemeinbildung auch spezifische Vorbildungen im kaufmännischen Bereich vermitteln. Das Fach BWL spielt in diesem berufsbezogenen Qualifizierungsprozess eine zentrale Rolle, so dass auch das pädagogische Handeln der betreffenden Lehrpersonen für ihre Schüler besonders relevant sein dürfte (Eder, 2004).

2 Der Verfasser dankt den Diplomandinnen Sabine Enzenebner, Sabrina Hanser, Sabine Häusler, Eva-Maria Hemetsberger, Michaela Disslbacher-Fink, Melitta Duschlbauer, Regina Peyrl, Renate Raab und Martina Voura für die Überlassung der Daten und Prof. Dr. Georg Hans Neuweg für die Zusammenarbeit bei der Konzeption und Organisation der Gesamtstudie.

\section{Literatur}

Baumert, J., Heyn, S. \& Köller, O. (1992). Das Kieler Lernstrategien-Inventar (KLI). Kiel: Institut für die Pädagogik der Naturwissenschaften an der Universität Kiel.

Bortz, J. \& Döring, N. (2003). Forschungsmethoden und Evaluation für Human- und Sozialwissenschaftler (3. Aufl.). Berlin: Springer.

Ditton, H. (2002). Lehrkräfte und Unterricht aus Schülersicht. Ergebnisse einer Untersuchung im Fach Mathematik. Zeitschrift für Pädagogik, 48, 262-286.

Doyle, W. (1986). Classroom organization and management. In M. C. Wittrock (Ed.), Handbook of research on teaching (3rd ed.) (pp. 392-431). New York: Macmillan.

Eder, F. (2004). Der Einfluss einzelner Lehrpersonen auf das Befinden von Schülerinnen und Schülern. In T. Hascher (Hrsg.), Schule positiv erleben. Ergebnisse und Erkenntnisse zum Woblbefinden von Schülerinnen und Schülern (S. 91-112). Bern: Haupt.

Emmer, E. T., Evertson, C. M., Sanford, J. P., Clements, B. S. \& Worsham, M. E. (1985). Classroom management for secondary teachers. Englewood Cliffs: Prentice-Hall.

Fartacek, W., Eder, F. \& Mayr, J. (1987). Schwierigkeiten von Lehrerstudenten und Lehrern im Umgang mit Schülern. Erziehung und Unterricht, 137, 12-24.

Gordon, T. (1997). Lehrer-Schüler-Konferenz (11. Aufl.). München: Heyne.

Gruehn, S. (2000). Unterricht und schulisches Lernen. Schüler als Quellen der Unterrichtsbeschreibung. Münster: Waxmann.

Helmke, A. (2004). Unterrichtsqualität-erfassen, bewerten, verbessern (3. Aufl.). Seelze: Kallmeyer.

Jones, V. F. \& Jones, L. S. (2004). Comprehensive classroom management. Creating communities of support and solving problems (7th ed.). Boston: Allyn and Bacon.

Kounin, J. S. (1970). Discipline and group management in classrooms. New York: Holt, Rinehart \& Winston.

Kunter, M., Schümer, G., Artelt, C., Baumert, J., Klieme, E., Neubrand, M., Prenzel, M., Schiefele, U., Schneider, W., Stanat, P., Tillmann, K.-J. \& Weiß, M. (2002). PISA 2000: Dokumentation der Erhebungsinstrumente. Materialien aus der Bildungsforschung, Nr. 72. Berlin: Max Planck-Institut für Bildungsforschung.

Lohmann, G. (2005). Mit Schülern klarkommen - Professioneller Umgang mit Unterrichtsstörungen und Disziplinkonflikten. 3. Aufl. Berlin: Cornelsen Scriptor.

Mayr, J. (2002). Mitarbeit und Störung im Unterricht: Klassenführung an HASCH und HAK. In P. Baumgartner \& H. Welte (Hrsg.), Reflektierendes Lernen. Beiträge zur Wirtschaftspädagogik (S. 35-50). Innsbruck: Studien-Verlag.

Mayr, J. (2004). Mitarbeit und Störung im Unterricht: Pädagogische Handlungsstrategien von Lehrern an höheren Schulen. Unser Weg, 59, 21-26.

Mayr, J., Eder, F. \&. Fartacek, W. (1991). Mitarbeit und Störung im Unterricht: Strategien pädagogischen Handelns. Zeitschrift für Pädagogische Psychologie, 5, 43-55.

Mayr, J., Eder, F. \& Fartacek, W. (2005). Linzer Diagnosebogen zur Klassenführung (LDK). [http://ius.uni-klu.ac.at/projekte/ldk/] 
Mayr, J. \& Neuweg, G. H. (2006). Der Persönlichkeitsansatz in der Lehrer/innen/forschung. Grundsätzliche Überlegungen, exemplarische Befunde und Implikationen für die Lehrer/innen/bildung. In U. Greiner \& M. Heinrich (Hrsg.), Schauen, was 'rauskommt. Kompetenzförderung, Evaluation und Systemsteuerung im Bildungswesen. (S. 183-206). Münster: Lit.

Rogers, C. R. (1969). Freedom to learn: A view of what education might become. Columbus, $\mathrm{OH}$ : Charles Merrill.

Rost, D. H. (2005). Interpretation und Bewertung pädagogisch-psychologischer Studien. Weinheim: Beltz.

Schreiber, B. (1998). Selbstreguliertes Lernen. Münster: Waxmann.

Stockinger, S. (1999). Störendes Schülerverhalten - Können Lehrer etwas dagegen tun? Diplomarbeit am Institut für Pädagogik und Psychologie der Universität Linz.

Tausch, R. \& Tausch, A.-M. (1998). Erziehungspsychologie. Begegnung von Person zu Person (11. Aufl.). Göttingen: Hogrefe.

Weinert, F. E. (Hrsg.). (1996). Psychologie des Lernens und der Instruktion. Enzyklopädie der Psychologie. Pädagogische Psychologie, Vol 2. Göttingen: Hogrefe.

Weinert, F. E. \& Helmke, A. (1996). Der gute Lehrer: Person, Funktion oder Fiktion? In A. Leschinsky (Hrsg.), Die Institutionalisierung von Lehren und Lernen (S. 223-233). Weinheim: Beltz.

Wendt, W. (2001). Belastung von Lehrkräften - Fakten zu Schwerpunkten, Strukturen und Belastungstypen. Eine repräsentative Befragung von Berliner Lehrerinnen und Lehrern. Landau: Empirische Pädagogik.

Schlagworte: Lehrer-Schüler-Beziehung, Unterricht

\section{Gestion de classe au secondaire II. Stratégies et modèles d'actions enseignantes réussies}

\section{Résumé}

Une enquête dans une haute école commerciale comprenant 75 professeurs et leurs étudiants, examine quelles stratégies de gestion de classe contribuent à ce que les étudiants travaillent bien et à un minimum de comportements dérangeants. Il ressort que l'organisation de l'enseignement, le fait de favoriser les relations sociales et le contrôle des comportements des étudiants jouent un rôle significatif. Le comportement des professeurs dont la réussite est notable se situe, selon la stratégie, dans une marge plus ou moins grande, l'enquête mettant à jour plusieurs modèles de gestion de classe réussie.

Mots clés: relation maître-élève, enseignement 


\section{Gestione della classe nelle scuole superiori: strategie e modelli per gli insegnanti}

\section{Riassunto}

In uno studio realizzato in scuole di commercio superiori che ha coinvolto 75 insegnanti con i loro studenti si è analizzato quali strategie di conduzione della classe favoriscono una buona partecipazione e un insegnamento meno gravato da interferenze. I risultati hanno mostrato delle caratteristiche specifiche attinenti alla gestione dell'insegnamento, al miglioramento delle relazioni sociali e del controllo del comportamento. Il comportamento dei buoni insegnanti si situa all'interno di margini che possono variare a seconda della strategia e che si articola secondo diversi modelli di conduzione.

Parole chiave: relazione allievo-docente, insegnamento

\section{Classroom Management in Higher Secondary Schools: Strategies and Patterns of Successful Teacher Behaviour}

\footnotetext{
Summary

A study of 75 business college teachers and their students was carried out to ascertain which classroom management strategies contribute to a large degree of cooperation and a minimum of disruptive behaviour. Both strategies of teaching, of fostering social relationships and of controlling students' behaviour appeared to be significant. The behavioural range of successful teachers lies within certain boundaries differing by the respective strategies and showing varying patterns of strategies.
}

Keywords: teacher-pupil-relation, teaching 